\title{
Nie sollst Du mich befragen ...
}

\author{
Maria-Theresia Schafmeister
}

Eingang des Beitrages: 10.6.2014 / Eingang des überarbeiteten Beitrages: 3.7.2014 / Online veröffentlicht: 23.8.2014

(C) Springer-Verlag Berlin Heidelberg 2014

Darum bat Lohengrin seine Elsa inständig. Sie tat es doch! Und Richard Wagner hat es in unvergleichlicher Weise vertont. Richard Wagner und Bayreuth, Bayreuth und die Fachsektion Hydrogeologie. Die Mitgliederversammlung der Fachsektion Hydrogeologie in der DGG ließ sich befragen - und zwar zu allerhand weitreichenden Entscheidungen.

Das Hauptthema der diesjährigen Mitgliederversammlung war die formale Beschlussfassung über die Eintragung der nunmehr seit 45 Jahren mit allen Merkmalen der Selbständigkeit agierenden Fachsektion Hydrogeologie in das Vereinsregister. Der beinahe wichtigere notwendige Beschluss galt der Satzung des Vereins, die mit einer neuen Präambel versehen (s. www.fh-dgg.de) aus unserer soliden bisherigen Geschäftsordnung entwickelt worden war.

Nie sollst Du mich befragen? Doch - aber vorher lieber Vorstand informiere mich! In den zwei Jahren, die seit der FH-Tagung in Dresden vergangen sind, hat sich der agierende Vorstand der FH-DGG tatkräftig unterstützt durch seinen Beirat mit dem Auftrag der Mitgliederversammlung beschäftigt, wie unserem Fach Hydrogeologie mehr Eigenständigkeit und Sichtbarkeit zu verschaffen wäre. Geprüft werden sollte ebenfalls, inwieweit dieses Ziel unter Beibehaltung des bisherigen rechtlichen Status der FH erreicht werden kann. Der Vorstand, das waren Martin Sauter/Uni Göttingen, Maria-Th. Schafmeister/Uni Greifswald, Thomas Himmelsbach/BGR Hannover, Thomas Schiedek/TU Darmstadt, Stefan Peiffer/Uni Bayreuth und David Kuntz/ tewag GmbH Starzach-Felldorf. In intensiver Zusammenarbeit mit der Geschäftsstelle der FH Frau Ruth KaufmannKnoke und schließlich unter juristischer Beratung durch

M.-T. Schafmeister $(\square)$

Lehrstuhl für Angewandte Geologie/Hydrogeologie, Greifswald, Deutschland

E-Mail: schaf@uni-greifswald.de
Herrn RA Kay-Uwe Lesueur hat der Vorstand eine transparente Informationspolitik betrieben und in zahlreichen Schreiben die Mitglieder der FH, also den Souverän unseres Vereins von seinen Schritten unterrichtet und die Entscheidungsgrundlagen dargelegt.

Nie sollst Du mich befragen? Doch, jetzt musste der Beschluss über die Eintragung der FH in das Vereinsregister erfragt werden. Und mit 98,46\%-iger Mehrheit hat sich die Mitgliederversammlung für diesen Schritt ausgesprochen. Der Vorstand der FH bedankt sich ausdrücklich für dieses große Vertrauen in seine Arbeit, vor allem der in den vergangenen zwei Jahren.

Aber hier dürfen und werden wir nicht innehalten. Jetzt erst recht muss vorangeschritten werden. Die Fachsektion Hydrogeologie - nach der formalen Eintragung in das Vereinsregister FH e. V. - hat nun viel zu tun: in erster Linie geht es um Hydro-Geologie, der wollten wir mit diesem Schritt mehr Sichtbarkeit und mehr Handlungsfreiheit verschaffen - national wie international. Die FH ist außerordentlich erfolgreich in ihren Fortbildungsveranstaltungen; können und wollen wir hier die hervorragende und gefragte hydrogeologische Expertise auch international weiter verbreiten? Wie binden wir unsere Mitglieder, die auch Mitglieder der IAH (International Association of Hydrogeologists) sind, besser an die FH an? In diesem Zusammenhang sei darauf hingewiesen, dass der 2. Stellvertretende Vorsitz der FH und die gleichzeitige Präsidentschaft der German Section of IAH von Thomas Himmelsbach auf Johannes Barth/ Uni Erlangen übergegangen ist. Thomas Himmelsbach gilt unser großer Dank für seine langjährige Arbeit!

Eine weitere Frage, die uns beschäftigen sollte, ist die Ausbildung in unserem Fach an den deutschen Universitäten! Wo sind starke hydrogeologische Standorte, wo verschwinden sie vielleicht? 
Ganz wichtig wird uns, dem Vorstand, aber die Aufgabe sein, uns weiterhin in der geologischen Fachwelt zu bewähren. Die enge Kooperation mit der Deutschen Gesellschaft für Geowissenschaften liegt uns allen besonders am Herzen. Wir sind Geologen und verstehen uns als die dem Wasser zugewandten Wissenschaftler, die die unverzichtbare geologische Kompetenz einbringen müssen. Sonst wird es niemand können! Hier, das weiß ich, spreche ich für alle meine Kollegen des alten und neu gewählten Vorstands der FH: Martin Sauter (danke für Deine moderaten Moderationen), Johannes Barth (viel Erfolg als Mittelsmann zur IAH), Thomas Schiedek (verrechne Dich bloß nicht, Schatzmeister), Stefan Peiffer (danke für die Übersicht beim Schriftführen) und David Kuntz (danke für die genialen Ideen, die in die Öffentlichkeit gehören). Einschließen kann ich die Geschäftsstelle, namentlich Frau Ruth Kaufmann-Knoke (danke für den Antrieb, den wir anderen immer brauchen werden).

Liebe Mitglieder der FH, lassen Sie uns nun gemeinsam wieder sehr viel, sehr gute Hydrogeologie machen - und die dann auch in unserer Grundwasser publizieren. Darauf freut sich.

Ihre

Maria-Th. Schafmeister (Vorsitzende der FH) GLÜCK AUF 